\title{
Determinantes da Demanda de Fertilizantes no Brasil no período de 1970 a 2002
}

\author{
Alexandre Chibebe Nicolella ${ }^{1}$ \\ Diogo S. Dragone ${ }^{2}$ \\ Carlos José Caetano Bacha ${ }^{3}$
}

Resumo: Este trabalho estima uma equação de demanda para fertilizantes no Brasil, considerando o período de 1970 a 2002. Para tanto, procedimentos de análise de co-integração são utilizados. Constatou-se que apenas o modelo em nível (estimativa pelos mínimos quadrados ordinários), válido para o longo-prazo, apresenta bons resultados econométricos. A demanda por fertilizantes é inelástica a variações de preços e ao volume de crédito rural, o que é coerente com as estimativas internacionais feitas sobre a demanda de fertilizantes e com a essencialidade que o fertilizante tem para a agricultura brasileira.

Palavras-chave: fertilizantes, consumo, modelos de co-integração

\section{Classificação JEL: Q1, Q11}

Abstract: This paper estimates an equation for fertilizer demand in Brazil, considering time period from 1970 to 2002. Co-integration analysis procedures have been used and they show that just the level model (LSO method), valid for long term, presents good econometrics results. Fertilizer demand is inelastic to both price fluctuations and rural credit supplied. These results

\footnotetext{
${ }^{1}$ Doutorando do Programa de Economia Aplicada - ESALQ/USP.

${ }^{2}$ Mestre em Economia Aplicada - ESALQ/USP.

${ }^{3}$ Professor Titular da ESALQ/USP.
} 
are compatible with the international estimates and they also confirm the importance of fertilizer as an input for the Brazilian agriculture.

Key words: fertilizer, demand, co-integration analysis

JEL Classification: Q1, Q11 (Agriculture - Aggregate Supply and Demand Analysis; Prices)

\section{Introdução}

O objetivo desse trabalho é analisar os determinantes da demanda por fertilizantes no Brasil no período de 1970 a 2002. Para tanto, um modelo econométrico é estimado e seus parâmetros são analisados. O período de 1970 a 2002 foi escolhido pela disponibilidade de informações e por ser uma fase de grande expansão do consumo de fertilizantes no país.

Os solos brasileiros possuem, em sua maioria, limitações de fertilidade natural, sendo que $84 \%$ desses solos possuem problemas com acidez (Amaral et al., 1999). Segundo Santos (1999), 40\% dos solos brasileiros são classificados como latossolos, possuindo características físicas excelentes e graves limitações quanto a sua fertilidade. Entretanto, Santos (1999) ressalta que os latossolos tornam-se altamente produtivos quando utilizados sobre manejo tecnificado.

O manejo tecnificado inclui, além de outras práticas ${ }^{4}$, o uso intensivo de fertilizantes. Esses são compostos de macronutrientes (especialmente nitrogênio, fósforo e potássio) e micronutrientes (boro, zinco, silício, entre outros), capazes de aumentar as características de fertilidade dos solos para os níveis exigidos pelos novos cultivares melhorados e mais produtivos.

A área cultivada no Brasil aumentou até o final da década de 1980. Após esse período, essa área seguiu tendência declinante. Entretanto, a produção mostra-se sempre crescente a uma taxa de $4,8 \%$ a.a. (considerando as 38 principais culturas) no período de 1970 a 2002. Essa mudança de estilo de crescimento da agricultura se deve a inovações adotadas sob a forma de: maior consumo de fertilizantes, melhoramento genético, novas técnicas de plantio, inovação mecânica, por exemplo.

\footnotetext{
${ }^{4}$ Algumas dessas práticas são: plantio direto, uso de corretivos, combate à erosão, entre outras.
} 
O consumo brasileiro de fertilizantes passou de 958 mil toneladas em 1970 para 7,77 milhões de toneladas em 2002 (Associação Nacional para Difusão de Adubos - ANDA). Tomando a área com lavouras, o consumo médio de fertilizantes por hectare passou de 18,5 kg em 1970 para $146 \mathrm{~kg}$ em 2002. Esse grande crescimento no consumo doméstico de fertilizantes fez o Brasil ser o 4o maior mercado mundial de fertilizantes, precedido pela China, EUA e Índia (Neves et al, 2002).

Apesar desse grande crescimento no consumo brasileiro de fertilizantes, pouco se conhece sobre seus determinantes. Isto motiva a realização deste trabalho.

O presente trabalho está organizado em sete seções, incluindo esta introdução. A seção 2 faz uma síntese da literatura internacional e nacional sobre modelos matemáticos e econométricos sobre a demanda de fertilizantes, ressaltando as estimativas geradas para a elasticidade-preço da demanda de fertilizantes. A partir dessa revisão é ressaltada a contribuição do presente trabalho. A seção 3 analisa a evolução do consumo de fertilizantes no Brasil, destacando seus períodos de expansão e redução. Essa evolução é associada à evolução dos preços de fertilizantes, ao crédito rural para a aquisição do produto e ao índice de preços recebidos pelos produtores, as quais são três das mais importantes variáveis determinantes do consumo de fertilizantes. A seção 4 apresenta a função demanda a ser estimada e os procedimentos econométricos seguidos. A seção 5 discute os dados utilizados. A seção 6 apresenta e discute os resultados obtidos na estimativa da equação de demanda de fertilizantes. Finalmente, a seção 7 apresenta as conclusões do trabalho.

\section{Revisão de literatura}

Há na literatura diversos trabalhos que usam diferentes métodos para estimar a demanda por fertilizantes e sua elasticidade preço. Burrell (1989) sintetiza alguns trabalhos, e parte desses estão descritos no Quadro 1. Constata-se que a literatura não é coincidente sobre a dimensão da elasticidade-preço da demanda de fertilizantes. No entanto, a maioria dos trabalhos indica que a demanda de fertilizantes é inelástica a preços, principalmente no curto-prazo. 
Quadro 1. Síntese de alguns trabalhos sobre estimativas da demanda de fertilizantes na Europa e EUA.

\begin{tabular}{|c|c|c|c|c|c|c|}
\hline Autor (es) & País analisado & $\begin{array}{l}\text { Abran- } \\
\text { gência }\end{array}$ & $\begin{array}{c}\text { Insu- } \\
\text { mo }\end{array}$ & $\begin{array}{c}\text { Metodologia } \\
\text { Utilizada }\end{array}$ & $\begin{array}{c}\text { Período de } \\
\text { Tempo }\end{array}$ & $\begin{array}{l}\text { Elasticidade } \\
\text { Preço }\end{array}$ \\
\hline Lingard(1971) & Reino Unido & Todo setor & $\mathrm{N}$ & ME/ES/ST & 1956-1958 & $\begin{array}{c}-1,089 \\
-0,60(\mathrm{cp}) \\
-1,18(\mathrm{lp}) \\
\end{array}$ \\
\hline England(1986) & Reino Unido & Cereais & $\mathrm{N}$ & PL & 1971-1981 & $\begin{array}{c}-0,08,-0,13 \\
-0,24\end{array}$ \\
\hline Burrell(1989) & Reino Unido & Todo setor & $\mathrm{F}$ & $\mathrm{ME} / \mathrm{Sis} / \mathrm{ST}$ & 1964-1985 & $-0,47$ a $-0,50$ \\
\hline Boyle(1981) & Irlanda & Todo setor & $\mathrm{F}$ & $\mathrm{ME} / \mathrm{Sis} / \mathrm{ST}$ & 1953-1977 & $-0,62$ \\
\hline Higgings(1986) & Irlanda & Todo setor & $\mathrm{F}$ & $\mathrm{ME} / \mathrm{Sis} / \mathrm{CS}$ & 1982 & $-1,38$ \\
\hline $\begin{array}{c}\text { Bonnieux e } \\
\text { Rainelli }\end{array}$ & França & Todo setor & $\mathrm{F}$ & ME/ES/ST & 1959-1984 & $\begin{array}{l}-0,33(\mathrm{cp}) \\
-1,10(\mathrm{lp}) \\
\end{array}$ \\
\hline Anker e Schmitz & Alemanha & Todo setor & $\mathrm{F}$ & $\mathrm{ME} / \mathrm{Sis} / \mathrm{ST}$ & 1964-1983 & $-0,19$ \\
\hline Dubgaard (1986) & Dinamarca & Todo setor & $\mathrm{N}$ & $\mathrm{ME} / \mathrm{ES} / \mathrm{ST}$ & 1956-1984 & $-0,19$ \\
\hline Ray(1982) & Estados Unidos & Todo setor & $\mathrm{F}$ & $\mathrm{ME} / \mathrm{Sis} / \mathrm{ST}$ & $1939-1977$ & $-0,4875$ \\
\hline Weaver(1983) & Estados Unidos & Todo setor & $\mathrm{F}$ & $\mathrm{ME} / \mathrm{Sis} / \mathrm{ST}$ & $1950-1970$ & $-1,377$ \\
\hline $\begin{array}{c}\text { Denblay e } \\
\text { Vroomen(1993) }\end{array}$ & Estados Unidos & Milho & $\mathrm{F}$ & ME/ES/ST & 1964-1989 & $\begin{array}{l}-0,21 \text { a }-0,25(\mathrm{cp}) \\
-0,31 \text { a }-0,41(\mathrm{lp})\end{array}$ \\
\hline $\begin{array}{c}\text { Mergos e } \\
\text { Stoforos(1997) }\end{array}$ & Grécia & Todo setor & $\mathrm{F}$ & ME/ES/ST & 1961-1993 & $\begin{array}{c}-0,36(\mathrm{cp}) \mathrm{e}- \\
\text { 0,81(lp) }\end{array}$ \\
\hline
\end{tabular}

Legenda: $\mathrm{N}=$ nitrogênio, $\mathrm{F}=$ todos fertilizantes

$\mathrm{ME}=$ modelo econométrico, $\mathrm{PL}=$ programação linear, $\mathrm{ES}=$ equação simples, Sis = sistema de equações, $\mathrm{ST}=$ dados séries temporais, $\mathrm{CS}=$ dados corte seccional

Fonte: Burrell (1989), Denblay e Vroomen(1993), Mergos e Stoforos(1997)

Para Denblay \&Vroomen (1993), os trabalhos apresentados no Quadro 1 podem ser divididos em duas categorias. A primeira inclui equações fortemente baseadas na teoria dual da função custo ou da função lucro. A segunda aproximação envolve a especificação do que pode ser entendida como equações na forma reduzida da maximização do lucro da propriedade agrícola. Ambas categorias de equações estão sujeitas a críticas.

Para Burrell (1989), as duas categorias de equações possuem problemas na estimação. Na primeira, a imposição teórica de equações simultâneas raramente possui aderência aos estudos empíricos. Além disso, o produto planejado é estimado sem considerar problemas climáticos como, por exemplo, secas e excesso de chuvas e outras variações residuais não relacionadas à expectativa de preço. Portanto, o produto planejado difere do produto observado.

A estimação do modelo tendo a segunda categoria como teoria, segundo Burrell (1989), pode levar a uma correlação espúria entre as variáveis se não for feita a análise de co-integração. 
No Quadro 2 são apresentados os trabalhos que estimaram a demanda de fertilizantes no Brasil.

Quadro 2. Síntese de alguns trabalhos sobre estimativas da demanda de fertilizantes no Brasil.

\begin{tabular}{|c|c|c|c|c|c|}
\hline Autor (es) & Abrangência & Insumo & $\begin{array}{c}\text { Metodologia } \\
\text { Utilizada }\end{array}$ & $\begin{array}{c}\text { Período de } \\
\text { Tempo }\end{array}$ & $\begin{array}{c}\text { Elasticidade } \\
\text { Preço }\end{array}$ \\
\hline \multirow{4}{*}{$\begin{array}{c}\text { Soares, Barros } \\
\text { e Carmo } \\
(1983)\end{array}$} & Brasil & $\mathrm{F}$ & $\mathrm{ME} / \mathrm{ES} / \mathrm{ST}$ & $1954-1979$ & $\begin{array}{c}-0,33 \text { a }-0,76 \\
\text { (cred) } \\
-0,55 \text { a -0,730 } \\
\text { (fert) }\end{array}$ \\
\cline { 2 - 6 } & Norte/Nordeste & $\mathrm{F}$ & $\mathrm{ME} / \mathrm{ES} / \mathrm{ST}$ & $1954-1979$ & $\begin{array}{c}-0,609 \text { (fert c/ } \\
\text { subsídio) }\end{array}$ \\
\cline { 2 - 6 } & Sul & $\mathrm{F}$ & $\mathrm{ME} / \mathrm{ES} / \mathrm{ST}$ & $1954-1979$ & $\begin{array}{c}-0,558 \text { (fert c/ } \\
\text { subsídio) }\end{array}$ \\
\cline { 2 - 6 } & São Paulo & $\mathrm{F}$ & $\mathrm{ME} / \mathrm{ES} / \mathrm{ST}$ & $1949-1971$ & $\begin{array}{c}-0,25 \text { (cp) } \\
-2,48(\mathrm{lp})\end{array}$ \\
\hline \multirow{2}{*}{$\begin{array}{c}\text { Cibantos } \\
(1972)\end{array}$} & São Paulo & $\mathrm{N}$ & $\mathrm{ME} / \mathrm{ES} / \mathrm{ST}$ & $1948-1972$ & $\begin{array}{c}-0,48(\mathrm{cp}) \\
-1,12(\mathrm{lp})\end{array}$ \\
\hline \multirow{2}{*}{$\begin{array}{c}\text { Pescarin } \\
(1974)\end{array}$} & São Paulo & $\mathrm{P}_{2} \mathrm{O}_{4}$ & $\mathrm{ME} / \mathrm{ES} / \mathrm{ST}$ & $1948-1972$ & $\begin{array}{c}-0,31(\mathrm{cp}) \\
-0,76(\mathrm{lp})\end{array}$ \\
\cline { 2 - 6 } & São Paulo & $\mathrm{K}_{2} \mathrm{O}$ & $\mathrm{ME} / \mathrm{ES} / \mathrm{ST}$ & $1948-1972$ & $\begin{array}{c}-0,30(\mathrm{cp}) \\
-0,60(\mathrm{lp})\end{array}$ \\
\hline
\end{tabular}

Legenda: $\mathrm{N}=$ nitrogênio, $\mathrm{F}=$ todos fertilizantes

$\mathrm{ME}=$ modelo econométrico, $\mathrm{ES}=$ equação simples, $\mathrm{ST}=$ dados séries temporais

As estimativas de elasticidade preço para a demanda de fertilizante no Brasil são semelhantes àquelas encontradas no exterior. Sendo que no curto prazo apresentam-se como sendo inelásticas. As estimativas de Soares, Barros e Carmo (1983) para a elasticidade preço da demanda do fertilizante para o Brasil variaram entre -0,33 e - 0,76. Para o Estado de São Paulo, Cibantos (1972) encontrou elasticidade preço de $-0,25$ no curto prazo e $-2,48$ no longo prazo. Desagregando o fertilizante em $\mathrm{N}, \mathrm{P}_{2} \mathrm{O}_{5}$ e $\mathrm{K}_{2} \mathrm{O}$, Pescarin (1974) obteve elasticidade preço no curto prazo variando de $-0,30$ a $-0,48$ e no longo prazo de $-0,60$ a $-1,12$.

Observa-se que os trabalhos apresentados no Quadro 2 só consideraram a demanda de fertilizantes até 1979. Grandes aumentos no consumo desse produto ocorreram nas décadas de 1980 e 1990, com situações diferentes, das vigentes na década de 1970, quanto a preço do fertilizante e disponibilidade de crédito para sua aquisição. Assim, o presente trabalho 
apresenta, como contribuição ao conhecimento disponível, a análise da demanda de fertilizantes para um período ainda não considerado pela literatura disponível.

\section{Evolução do consumo de fertilizantes no Brasil}

Levando em conta os dados da Associação Nacional para a Difusão de Adubos (ANDA), é interessante registrar que o crescimento do consumo de fertilizantes no mercado interno foi de aproximadamente $680 \%$ em 32 anos, passando de 998 mil toneladas em 1970 para 7,77 milhões de toneladas em 2002. Este crescimento não foi contínuo ao longo do tempo, apresentando reduções nos seguintes períodos (Gráfico 1): 1981 a 1983, 1988, 1989 e 1995, além de ter sido estagnado nos anos de 1997 a 1999. Estas interrupções no crescimento do consumo de fertilizantes se explicam por alterações em algumas variáveis que o afetam, em especial o preço do fertilizante e o crédito rural. Esses dois aspectos são analisados a seguir.

Gráfico 1. Brasil: consumo total de fertilizantes em milhões de toneladas, 1970-2002.

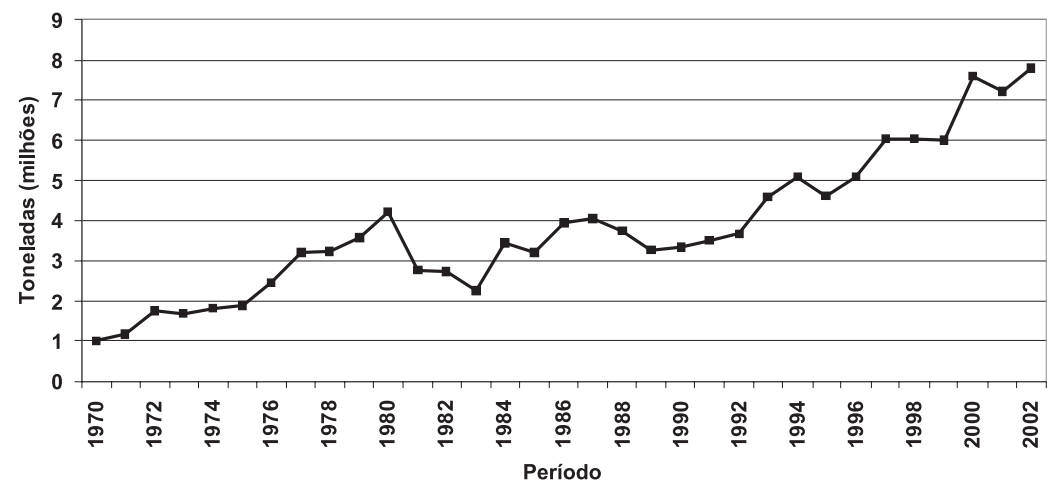

Fonte: IBGE/ANDA

\subsection{Evolução do Preço do Fertilizante}

O preço em moeda nacional do fertilizante é afetado por diversas variáveis, destacando-se: a cotação internacional do produto, a taxa de câmbio e os impostos e subsídios incidentes. 
Há tendência clara de declínio dos preços dos fertilizantes no período de 1975 a 2002 (Gráfico 2), após a forte alta ocorrida de 1972 a 1974. Essa elevação, possivelmente, é explicada pelo 1ํ choque do petróleo, pois alguns componentes dos fertilizantes são derivados do mesmo. A taxa de câmbio também tem efeitos nos preços dos fertilizantes, pois parte desses fertilizantes é importada. Na década de 1990, especialmente antes de 1999, a valorização cambial contribuiu para a redução do preço deflacionado em reais dos fertilizantes. A desvalorização cambial ocorrida a partir de 1999 explica, em parte, o pequeno aumento da cotação em moeda nacional do fertilizante.

Gráfico 2. Evolução dos Preços Ponderado da tonelada de Fertilizantes em mil reais de 2003 , período 1970 a 2002 .

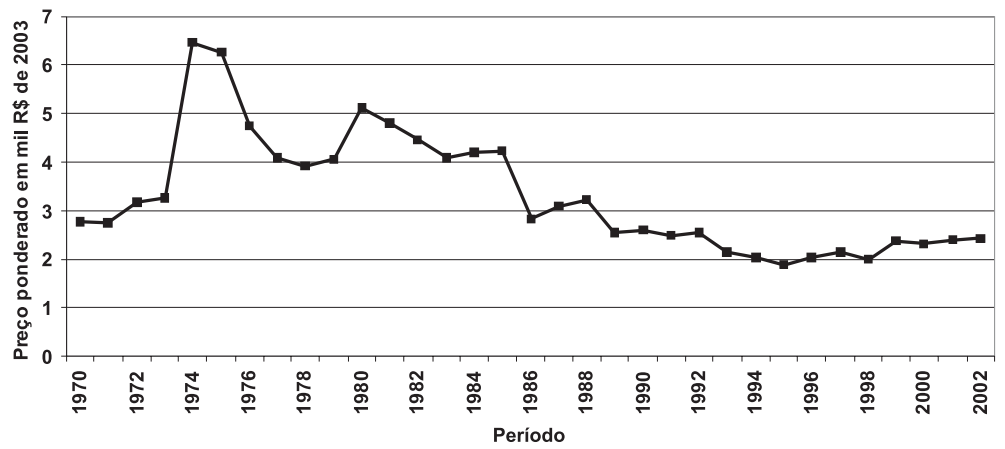

Fonte: Elaborada pelos autores

Nota: O preço ponderado é a média dos preços dos nutrientes ponderados pela sua participação no consumo aparente de fertilizantes em nutrientes.

O preço mostra-se negativamente correlacionado com o consumo, sendo de -0,54 o cálculo dessa correlação para o período de 1970 a 2002 e de $-0,59$ para o período de 1980 a 2002. Essa relação negativa entre preço e consumo de fertilizante é o que se espera como sendo normal.

\subsection{Crédito Rural para a compra de fertilizantes}

As primeiras tentativas de se criar uma política de crédito rural no Brasil ocorreram no início do século passado, com o crescimento das lavouras cafeeiras. No entanto, medidas concretas só foram implementadas em 1937, quando foi criada a Carteira de Crédito Agrícola e Industrial 
(CREAI) do Banco do Brasil e foi publicada a Lei no 492 regulamentando o Penhor Rural, que havia sido introduzido na legislação brasileira em 1885 (Lei no 3.272). No entanto, este tipo de crédito só passou a ser expressivo a partir da criação do Sistema Nacional de Crédito (SNCR) em 1965.

A partir dos anos 1950, o setor rural passou por um período de transformações e reestruturações, buscando não somente o crescimento extensivo da produção agrícola, mas também a modernização de sua base técnica (Guilhoto et al., 2000). A institucionalização do crédito rural no Brasil e a fixação de um piso de obrigatoriedade de empréstimo à agricultura sobre os depósitos à vista do sistema bancário permitiram uma forte expansão do crédito rural até o final dos anos 1970 (Gráfico 3). O crescimento médio do volume das aplicações de recursos de crédito rural, em termos reais, foi de $16,7 \%$ a.a. no período de 1969 a 1979. Em 1970, o crédito rural correspondia a $54 \%$ do produto do setor primário (Kageyama et al., 1990). Este crescimento foi impulsionado pela forte presença do setor público e pela aplicação compulsória dos bancos. A partir dos anos 1980, caracterizados por desequilíbrios macroeconômicos, houve forte redução do volume de crédito oficial.

Gráfico 3. Evolução do crédito custeio no Brasil em bilhões de reais de 2003, período 1970 a 2002.

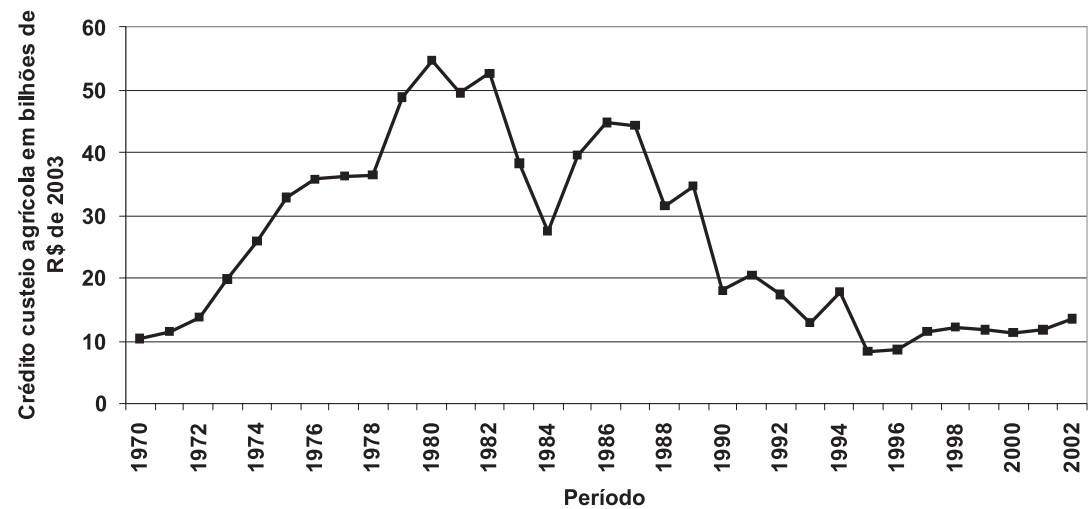

Fonte: Banco Central do Brasil - Anuário do Crédito Rural

A década de 1990 foi marcada por forte redução do nível de crédito de custeio disponível, marcando uma virada no sistema de financiamento agrícola nacional. Este novo sistema de financiamento é caracterizado 
pela maior participação das instituições privadas no financiamento de seus clientes (produtores), com empréstimos ressarcidos com recebimento da produção, gerando, por exemplo, a tão conhecida "Soja Verde". Empresas multinacionais financiam a aquisição de adubos, sementes e outros insumos necessários à realização da produção. Esta transformação é evidenciada com os dados do Gráfico 3, onde o crédito de custeio passou do nível médio de R \$ 34 bilhões por ano entre 1970 e 1989 para R\$ 13,5 bilhões por ano nos anos 1990 (a preços reais de 2003).

A correlação entre o consumo de fertilizante e o crédito de custeio foi baixa e negativa, $-0,35$, no período de 1970 a 2002 . Ao analisar essa correlação em dois sub períodos, de 1970 a 1980 e 1981 a 2002, encontra-se alta correlação positiva para o primeiro período, 0,94 e negativa para o segundo período -0,70. Esses valores mostram a essencialidade dos fertilizantes para a agricultura. Mesmo em fase de volumes decrescentes de crédito de custeio oficial (como o período de 1981 a 2002), o consumo de fertilizantes continuou a crescer. Isto foi possível devido ao uso de outras fontes de recursos (como os recursos próprios dos agricultores e créditos não oficiais fornecidos por empresas industriais e de comercialização).

\subsection{Outras Variáveis Afetando o Consumo de Fertilizante}

Além do preço do fertilizante e do volume de crédito de custeio, duas outras variáveis podem ser consideradas como determinantes do consumo de fertilizantes: a área cultivada com lavouras e um indicador de preços recebidos pelos produtores agrícolas.

O que se espera, a princípio, é que o aumento da área cultivada com lavouras implique aumento no consumo de fertilizantes. De fato, entre 1970 e 1980 há aumento da área cultivada com as 38 principais lavouras e aumento no consumo de fertilizantes (ver Gráfico 1). No entanto, a partir de 1990 há declínio na área cultivada com lavouras no Brasil, sendo que o consumo de fertilizantes continua a aumentar. $\mathrm{O}$ que se observa a partir de 1990 é que a produtividade da terra tem aumentado a um ritmo que permite a ampliação da produção agrícola com menor uso de terra. Esse aumento da produtividade da terra se faz, em parte, devido ao maior uso de fertilizantes. Esse comportamento distinto da relação 
entre área com culturas e consumo de fertilizante nos períodos de 1970 a 1989 e de 1990 a 2002 pode inviabilizar o uso da área de lavouras como variável determinante da demanda de fertilizantes.

Pode-se pensar que um índice de preços recebidos (IPR) seja também um dos determinantes do consumo de fertilizantes. Espera-se que o aumento do IPR gere estímulo ao consumo de fertilizantes, pois os produtores poderiam produzir maior quantidade de produtos agrícolas a serem vendidos a preços unitários maiores. Ao analisar a correlação entre consumo de fertilizante e IPR no período de 1970 a 2002 obtém-se o valor de 0,58 (demonstrando ser o IPR uma variável relevante para o entendimento da demanda por fertilizante). Considerando o período de 1970 a 1980, a correlação entre consumo de fertilizante e o IPR chega a 0,90 e para 1990 a 2002 tem-se o valor de 0,77.

No Gráfico 4 observa-se uma tendência de crescimento do índice de preços recebido (IPR) no período de 1970 a 2002. No entanto, chama atenção a forte queda entre os períodos de 1980 a 1982 e de 1986 a 1989. Parte dessas quedas pode ser explicada pelo alto índice inflacionário no período e pelos diversos planos que tentaram combater a escalada inflacionária.

Gráfico 4. Evolução do Índice de Preço Recebido (IPR) em reais de 2003, período 1970 a 2002.

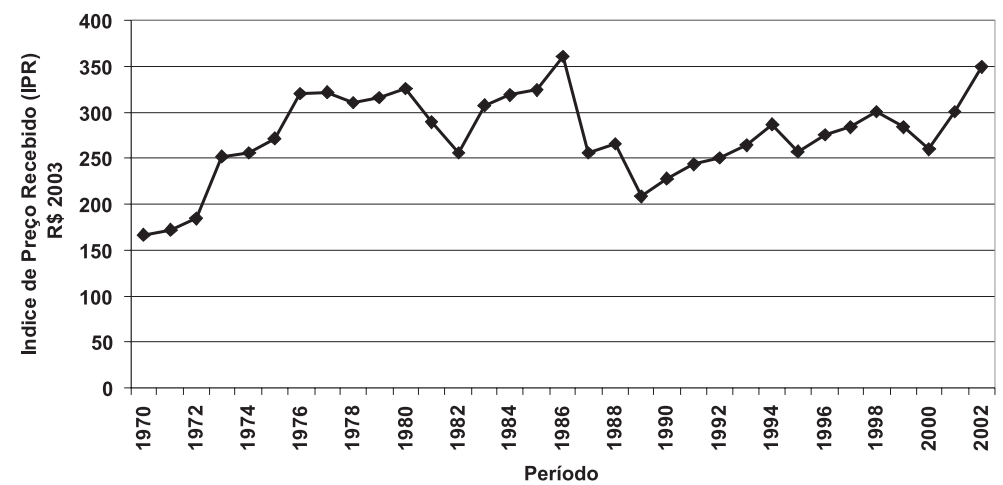

Fonte: Fundação Getulio Vargas (FGV).

Nota: base agosto de 1994 e deflacionado para dezembro de 2003 


\section{Modelo Econométrico}

Baseada na exposição da seção 3, a primeira equação de demanda a ser estimada é:

$$
\begin{aligned}
& \text { Cons }=f\left(P_{\text {Fert }}, C_{\text {cred }}, \text { Area, } I P R\right) \\
& \text { Onde } \\
& \text { Cons }=\text { consumo de fertilizantes } \\
& P_{\text {Fert }}=\text { preço do fertilizante } \\
& C_{\text {cred }}=\text { crédito de custeio } \\
& \text { Area = área cultivada com as } 38 \text { principais lavouras } \\
& I P R=\text { índice de preços recebidos }
\end{aligned}
$$

As variáveis foram logaritimizadas. Assim, obtêm-se diretamente as elasticidades marshalianas (para maiores detalhes ver Burrel 1989).

A equação (1) será, inicialmente, estimada pelo método dos mínimos quadrados ordinários (MQO) utilizando as variáveis em nível ${ }^{5}$. Este resultado, no entanto, pode gerar relações espúrias caso as variáveis não sejam co-integradas. Mas se as variáveis forem co-integradas, os resultados das estimativas via o MQO permitem avaliar as dinâmicas de longo-prazo (Maddala, 1988, p. 217).

A ordem de integração de uma variável é definida por Engle e Granger (1987, p.252). Segundo esses autores, “uma série sem componentes determinísticos, a qual possui uma representação ARMA, estacionária e irreversível após diferenciar d vezes, é dita ser integrada de ordem d, denotado por $\mathrm{X}_{\mathrm{t}} \sim \mathrm{I}(\mathrm{d})$ ”. A ordem de integração (d) é o número de vezes que uma variável precisa ser diferenciada para atingir a estacionariedade.

Para o teste da estacionariedade das séries utilizou-se o teste de Dickey e Fuller Aumentado (ADF), proposto por Dickey e Fuller (1981), que tem como base a seguinte regressão estimada por mínimos quadrados ordinários:

$$
\begin{aligned}
& \Delta X_{t}=\alpha+\beta t+\eta X_{t-1}+\sum_{i=1}^{p-1} w_{i} \Delta X_{t-i}+e_{t}, \text { com; } \\
& \eta=\sum_{i=1}^{p} \rho_{i}-1, \quad w_{i}=-\sum_{j=i+1}^{p} \rho_{i},=\text { drift, } t=\text { tendência e } p=\text { ordem do }
\end{aligned}
$$

processo auto regressivo que descreve o comportamento da série.

\footnotetext{
${ }^{5}$ Foram utilizados os pacotes econométricos Stata e Rats para as estimações
} 
Sendo, neste caso, a presença de raiz unitária testada pela hipótese $\mathrm{H}_{0}: \eta=0$. Os valores críticos estão tabulados em Dickey e Fuller (1981) e sua análise segue o procedimento proposto por Enders (1995). Os procedimentos para definição do processo auto-regressivo de ordem $p$ são os de AIC (Akaike) e o de SB (Schwarz) ${ }^{6}$.

No caso de haver duas variáveis $\mathrm{X}_{\mathrm{t} 1}$ e $\mathrm{X}_{\mathrm{t} 2}$ integradas de mesma ordem $d$ e que possuam relação linear estável de longo prazo (ou seja, $\mathrm{z}_{\mathrm{t}}=$ $\mathrm{X}_{\mathrm{t} 1}-\alpha \mathrm{X}_{\mathrm{t} 2}$, sendo $\mathrm{z}_{\mathrm{t}}$ estocástica e estácionária), diz-se que essas variáveis são co-integradas. Nesse caso, há necessidade de se utilizar um modelo de correção de erro.

Segundo Engle e Granger (1987, p.253) “os componentes do vetor $X_{t}$ são co-integrados de ordem $d, b$, denotado por $X_{t} \sim C I(d, b)$, se, i) todos os componentes de $X_{t}$ são I(d); ii) existe um vetor $\alpha(\alpha 0)$ onde $\mathrm{zt}=$ $\alpha^{\prime} X_{t} \sim \mathrm{I}(\mathrm{d}-\mathrm{b}), \mathrm{b}>0$. O vetor $\alpha$ é chamado de vetor de co-integração”.

Aplicou-se a metodologia de Johansen para testar a co-integração das séries. Tal metodologia é indicada quando houver mais de um vetor de co-integração e/ou existir endogeneidade do regressor. Caso haja a rejeição de $\mathrm{H}_{0}$ (ou seja, não há co-integração), é estimado o modelo nas diferenças incluindo os vetores de co-integração. Assim, pode-se analisar duas dinâmicas: i) de longo prazo, representada pelo modelo em nível e; ii) de curto prazo, representado pelo modelo nas diferenças incluindo os vetores de co-integração (Maddala, citado por Bacchi, 1994).

\section{Os dados utilizados}

Os dados utilizados nas regressões estimadas foram retirados de cinco fontes distintas, as quais são:

i) Anuário da Associação Nacional para Difusão de Adubos - ANDA;

ii) Anuário do Instituto Brasileiro de Geografia e Estatística - IBGE;

iii) Anuário Estatístico do Crédito Rural - BACEN;

${ }^{6}$ AIC: $\ln \hat{\sigma}^{2}+(2 / T)$ e SB: $\ln \hat{\sigma}^{2}+(\ln T / T)$. Sendo $\hat{\sigma}^{2}$ a soma dos quadrados dos resíduos de $X_{t}=X_{t-1}+\ldots+{ }_{p} X_{t-p}+e_{t}$ e T o número de observações. Para nosso estudo, partimos de uma especificação geral de 10 defasagens. A escolha de p é feita utilizando-se o menor valor para os critérios AIC e SB 
iv) Instituto de Economia Agrícola do Estado de São Paulo - IEA/ SAA/SP, e;

v) Fundação Getulio Vargas - FGV.

A Tabela 1 apresenta os dados utilizados nas regressões.

Os dados de consumos foram extraídos dos anuários do IBGE e ANDA, somando-se o consumo aparente de $\mathrm{N}, \mathrm{P}_{2} \mathrm{O}_{5}$ e $\mathrm{K}_{2} \mathrm{O}$.

A série de preços foi elaborada a partir dos preços correntes do nitrocálcio, superfosfato simples e cloreto de potássio, extraídos do banco de dados do IEA/SAA-ESP. Esses preços foram deflacionados para reais de dezembro de $2003^{7}$ e convertidos pelas respectivas concentrações em $\mathrm{N}, \mathrm{P}_{2} \mathrm{O}_{5}$ e $\mathrm{K}_{2} \mathrm{O}$. Com isso, obtém-se o preço do nutriente em reais de dezembro 2003 por tonelada. Para estabelecer um preço único para o fertilizante, utilizou-se o consumo aparente de cada nutriente como fator de ponderação.

Tabela 1 - Evolução do consumo de fertilizante em toneladas, do índice de preço em R\$ e do crédito custeio em milhão de R\$ para o Brasil, 1970 a 2002.

\begin{tabular}{|c|c|c|c|c|c|}
\hline Ano & $\begin{array}{l}\text { Consumo de } \\
\text { Nutrientes } \\
\text { (em toneladas) }\end{array}$ & $\begin{array}{c}\text { Preço } \\
\text { ponderado da } \\
\text { tonelada de } \\
\text { fertilizante } \\
(\mathrm{R} \$)^{*}\end{array}$ & $\begin{array}{l}\text { Crédito de } \\
\text { Custeio } \\
\text { Milhão (R\$)* }\end{array}$ & $\begin{array}{c}\text { Área Colhida } \\
\text { das } 38 \\
\text { principais } \\
\text { culturas } \\
\text { (em hectares) }\end{array}$ & $\begin{array}{l}\text { Índice de } \\
\text { preços } \\
\text { recebidos } \\
\text { (IPR) }\end{array}$ \\
\hline 1970 & 998.566 & 2.777 & 10.307 & 31.739 .861 & 165,978 \\
\hline 1971 & 1.165 .034 & 2.738 & 11.569 & 37.624 .692 & 172,356 \\
\hline 1972 & 1.746 .773 & 3.159 & 13.686 & 38.524 .322 & 184,498 \\
\hline 1973 & 1.679 .146 & 3.266 & 19.720 & 37.075 .571 & 251,403 \\
\hline 1974 & 1.824 .636 & 6.448 & 25.792 & 40.508 .176 & 255,809 \\
\hline 1975 & 1.894 .206 & 6.244 & 32.799 & 42.231 .589 & 271,059 \\
\hline 1976 & 2.464 .884 & 4.739 & 35.653 & 43.510 .242 & 319,914 \\
\hline 1977 & 3.185 .716 & 4.076 & 36.277 & 46.051 .443 & 322,152 \\
\hline 1978 & 3.222 .386 & 3.901 & 36.393 & 45.753 .252 & 309,868 \\
\hline 1979 & 3.567 .039 & 4.047 & 48.753 & 46.949 .990 & 316,189 \\
\hline 1980 & 4.200 .619 & 5.119 & 54.659 & 48.592 .943 & 325,967 \\
\hline 1981 & 2.753 .729 & 4.792 & 49.483 & 47.758 .784 & 289,351 \\
\hline 1982 & 2.718 .470 & 4.459 & 52.512 & 50.194 .927 & 256,422 \\
\hline
\end{tabular}

${ }^{7}$ Utilizou-se para o deflacionamento o Índice Geral e Preços - Disponibilidade Interna (IGP-DI) da Fundação Getúlio Vargas. 


\begin{tabular}{llllll}
1983 & 2.272 .033 & 4.097 & 38.167 & 44.362 .827 & 307,968 \\
1984 & 3.454 .508 & 4.213 & 27.394 & 48.785 .554 & 318,364 \\
1985 & 3.198 .055 & 4.236 & 39.539 & 50.763 .969 & 325,006 \\
1986 & 3.953 .397 & 2.832 & 44.815 & 52.537 .025 & 361,182 \\
1987 & 4.049 .645 & 3.087 & 44.333 & 52.490 .292 & 255,615 \\
1988 & 3.743 .748 & 3.229 & 31.529 & 54.400 .086 & 266,021 \\
1989 & 3.269 .730 & 2.531 & 34.498 & 54.214 .999 & 208,564 \\
1990 & 3.326 .275 & 2.603 & 18.000 & 49.563 .023 & 228,032 \\
1991 & 3.508 .722 & 2.475 & 20.425 & 49.664 .116 & 243,616 \\
1992 & 3.674 .530 & 2.531 & 17.239 & 49.712 .828 & 250,298 \\
1993 & 4.588 .997 & 2.145 & 12.805 & 45.667 .113 & 264,986 \\
1994 & 5.080 .208 & 2.035 & 17.700 & 50.351 .971 & 286,406 \\
1995 & 4.603 .812 & 1.878 & 8.205 & 49.763 .960 & 257,512 \\
1996 & 5.090 .885 & 2.019 & 8.535 & 44.646 .669 & 275,002 \\
1997 & 6.034 .573 & 2.143 & 11.350 & 46.525 .580 & 284,275 \\
1998 & 6.039 .886 & 2.003 & 12.063 & 45.760 .747 & 300,647 \\
1999 & 5.999 .957 & 2.374 & 11.726 & 47.942 .896 & 284,251 \\
2000 & 7.569 .857 & 2.302 & 11.197 & 49.088 .829 & 260,054 \\
2001 & 7.205 .000 & 2.403 & 11.715 & 49.231 .694 & 300,083 \\
2002 & 7.773 .000 & 2.432 & 13.444 & 52.078 .577 & 349,169 \\
\hline
\end{tabular}

Fonte: dados brutos coletados do BACEN, ANDA, IBGE, IEA, FGV.

Nota: *Valores convertidos em R \$ dezembro de 2003 pelo Índice Geral de Preços - Disponibilidade Interna (IGP-DI) da Fundação Getúlio Vargas.

A série de crédito de custeio foi extraída diretamente do Anuário Estatístico do Crédito Rural - BACEN e deflacionada para 2003.

O índice de preços recebidos (IPR) foi elaborado pela Fundação Getulio Vargas (FGV) tendo como base o mês de agosto de 1994 e deflacionado para dezembro de 2003.

A variável área foi extraída do Instituto Brasileiro de Geografia e Estatístico (IBGE) utilizando-se a soma da área colhida das 38 principais culturas ${ }^{8}$.

\footnotetext{
${ }^{8}$ Essas culturas são: abacate, algodão-arbóreo, algodão-herbáceo, alho, amendoim, arroz, aveia, batata, cacau, café, cana, castanha, cebola, centeio, cevada, coco, feijão, fumo, goiaba, guaraná, juta, laranja, limão, maçã, malva, mamona, mandioca, manga, maracujá, milho, pimenta, sisal, soja, sorgo, tangerina, tomate, trigo e uva.
} 


\section{Resultados}

Para estimar a equação (1) foi introduzida uma variável dummy ${ }^{9}$ para captar melhor a mudança na evolução do consumo de fertilizantes a partir da década de 1990. A estimativa pelo MQO da equação (1) com a variável dummy não evidenciou bom ajuste econométrico, com insignificância estatística para os coeficientes de $P_{\text {Fert }}$ e $C_{\text {Cred }}$ e com sinal negativo para os coeficientes $C_{\text {cred }}$, o qual não era esperado. Há indícios de forte multicolinearidade nessa estimativa, pois o modelo mostra-se altamente significativo (teste $\mathrm{F}$ ), sendo que a maior parte das suas variáveis explicativas não o são (pelo teste t).

Apesar do coeficiente estimado da variável Area ter sido positivo e estatisticamente significativo, constatou-se que essa variável é bastante determinada pelas demais, indicando que provavelmente a variável Area é a causadora do fenômeno da multicolinearidade. Além disso, o comportamento da área foi estável nos últimos anos e o consumo de fertilizante teve forte crescimento.

Prosseguindo, estima-se o modelo (1), por MQO, excluindo-se a variável Area. Os resultados estão apresentados na Tabela 2. Nota-se que não há indícios de multicolinearidade nesse modelo, sendo que as variáveis estimadas e o modelo são estatisticamente significativos ao nível de $10 \%$ e $5 \%$, expressos pelo teste t e teste $\mathrm{F}$, respectivamente.

Tabela 2 - Resultado do modelo de demanda por fertilizantes estimado em nível

\begin{tabular}{lccc}
\hline Variável & Coeficiente & Teste $\mathbf{~}$ & Pr $>$ [t] \\
Intercepto & 7,223 & $-3,13$ & 0,004 \\
$\mathbf{P}_{\text {Fert }}$ & $-0,655$ & 1,82 & 0,004 \\
$\mathbf{C}_{\text {Cred }}$ & 0,250 & 4,28 & 0,080 \\
IPR & 1,335 & $-3,07$ & 0,000 \\
Dummy & $-0,542$ & 3,10 & 0,005 \\
\hline $\mathbf{R}^{2}=0,671$ & $\mathbf{F}=32,05$ & $\mathbf{P r}>\mathbf{F}=<0,0001$ & $\mathbf{D W}=1,58$ \\
\hline
\end{tabular}

Fonte: dados da pesquisa

Os sinais comportam-se como esperado, ou seja, os coeficientes das variáveis preços do fertilizante e da dummy são negativos e os coeficientes das variáveis crédito de custeio e IPR são positivos. Por meio do teste DW não foi diagnosticado autocorrelação serial entre os resíduos.

${ }^{9}$ Para 1970 a 1990 considerou 1, e a partir de 1991 a dummy tem valor nulo. 
Os coeficientes apresentados na Tabela 2 equivalem às elasticidades marshalianas de longo prazo. Constata-se que a demanda de fertilizantes é inelástica a variações de preços. O aumento de $1 \%$ no preço do fertilizante diminui a demanda desse produto em 0,655\%. A demanda de fertilizantes é inelástica à variação do crédito de custeio. $\mathrm{O}$ aumento de $1 \%$ no volume de crédito de custeio aumenta a demanda por fertilizantes em 0,250\%. Mas a demanda de fertilizante é bastante sensível à variação do IPR. O aumento de $1 \%$ no IPR aumenta a demanda por fertilizante em $1,34 \%$

Os valores aqui encontrados possuem similaridades com os valores encontrados na literatura. Para Mergos e Stoforos (1997), a elasticidade preço da demanda por fertilizantes de longo prazo na Grécia foi $-0,81$. Para Denbaly e Vroomen (1993) essa elasticidade para os EUA variou entre -0,31 a -0,41. No Reino Unido, Burrel (1986) estimou elasticidade preço da demanda de fertilizantes de longo prazo entre $-0,47$ a $-0,50$.

Procedeu-se, então, ao teste de raiz unitária e constatou-se que a área é estacionária I(0); as variáveis $P_{\text {Fert }}, C_{\text {cred }}, I P R$ são estacionárias na primeira diferença, ou seja, I(1).

Pelo motivo descrito anteriormente e visando considerar variáveis estacionárias na mesma diferença e realizar teste de co-integração, o modelo foi simplificado, retirando a variável independente Area.

Na Tabela 3 está o teste de Dicker e Fuller Aumentado para as séries de consumo de fertilizantes, preço do fertilizante, crédito custeio e índice de preço recebido. Conclui-se que as séries são integradas de ordem 1, I(1). Em seguida, analisou-se a co-integração das séries utilizando-se a metodologia de Johansen, conforme descrito anteriormente. Foi estimada a presença de dois vetores de co-integração, o que leva a rejeitar a hipótese inicial de não co-integração das séries. 
Tabela 3 - Testes de raiz unitária para a variável consumo de fertilizante (Cons), preço de fertilizante (Pfer), crédito custeio (Ccred), com as variáveis na primeira diferença e as variáveis na segunda diferença.

\begin{tabular}{ccccccc}
\hline \multirow{2}{*}{ Variável } & \multicolumn{9}{c}{ Modelo 1* } & Modelo 2** \\
\cline { 2 - 7 } & $\tau_{\tau}$ & $\tau_{\beta \tau}$ & $\tau_{\mu}$ & $\tau_{\alpha \mu}$ & $\tau$ & $\tau$ \\
\hline Cons & $-3,19$ & 2,53 & $-1,95$ & 2,03 & 2,09 & $-5,59^{*}$ \\
\hline Pfer & $-2,81$ & $-2,49$ & $-1,37$ & 1,36 & $-0,19$ & $-5,51^{*}$ \\
\hline$C_{\text {Cred }}$ & $-2,54$ & $-2,56$ & $-1,46$ & 1,46 & 0,14 & $-5,95^{*}$ \\
\hline Area & $-2,77$ & 0,29 & $-3,42$ & 3,43 & 1,68 & $-5,59^{\mathrm{A}}$ \\
\hline IPR & $-2,76$ & 0,13 & $-2,85$ & 2,89 & 0,98 & $-5,71^{*}$ \\
\hline
\end{tabular}

Fonte: Dados de pesquisa

Notas: * Rejeita-se a hipótese de raiz unitária ao nível de $1 \%$ [valores críticos em Fuller (1976) e Dickey-Fuller (1981)]

Não houve autocorrelação serial baseado no teste Q de Ljung-Box.

O valor de $p$ usado, obtido pelos critérios de AIC e SB, foram de 9 para a variável consumo de fertilizantes e de 1 para as variáveis preço do fertilizante e crédito custeio.

A) Serie estacionária (I(0))

Modelo $1 \Rightarrow \Delta x_{t}=\alpha+\beta t+\gamma x_{t-1}+\sum_{i=1}^{p-1} \lambda_{i} \Delta x_{t} .+\varepsilon$. , nas versões com constanteetendência, sem tendência e sem tendência e constante. Modelo $2 \Rightarrow \Delta x_{t}=\gamma \Delta x_{t-1}+\sum_{i=1}^{p-2} \lambda_{i} \Delta x_{t-i}+\varepsilon_{t}$
definido após constatado a não existência de termos deterministas.

Para analisar a dinâmica de curto prazo, estima-se o modelo de correção de erro. Esse modelo é estimado com as variáveis diferenciadas incluindo um vetor de co-integração estimado anteriormente ${ }^{10}$. Os resultados estão apresentados na Tabela 4.

Tabela 4 - Resultado do modelo de correção de erro para a demanda por fertilizantes

\begin{tabular}{lccc}
\hline Variável & Coeficiente & Teste $t$ & $\mathbf{P r}>[\mathbf{t}]$ \\
\hline Intercepto & 0,921 & 1,14 & 0,264 \\
$\Delta$ PFert & $-0,150$ & $-0,64$ & 0,525 \\
$\Delta$ IPR & 0,230 & 0,88 & 0,387 \\
$\Delta$ Cred & 0,090 & 0,75 & 0,458 \\
Dummy & $-0,016$ & $-0,26$ & 0,800 \\
\hline $\mathbf{R} 1^{*}$ & 0,368 & 1,07 & 0,296 \\
\hline $\mathbf{R}^{2}=0,10$ & $\mathbf{F}=0,58$ & $\mathbf{P r}>\mathbf{F}=0,7154$ & $\mathbf{D W}=2,58$ \\
\hline
\end{tabular}

Fonte: dados da pesquisa

Nota: *R1 é o vetor de co-integração

Os sinais dos coeficientes comportam-se como esperado, mas o valor do teste $t$, para todas as variáveis, não se mostrou estatisticamente significativo

${ }^{10} \mathrm{O}$ segundo vetor não se mostrou significativo, justificando assim a sua retirada. 
a 10\% . Assim, conclui-se que não há relações de curto prazo entre a variável predita (demanda de fertilizante) e as preditoras, ou seja, as variáveis aqui propostas não explicam no curto prazo a demanda por fertilizante.

\section{Conclusões}

Este trabalho estimou a equação de demanda de fertilizantes no Brasil no período de 1970 a 2002. As variáveis independentes consideradas foram o preço do fertilizante, o crédito de custeio, área e o índice de preço recebido.

A estimativa do modelo de demanda de fertilizantes pelo método dos mínimos quadrados ordinários mostrou resultado melhor quando se exclui a área como variável explicativa. Isto se justifica pelo fato da agricultura brasileira, a partir de 1989, estar expandindo-se com menor uso da área. Isto só é possível, em parte, porque a produtividade da terra está aumentando, o que se deve, em parte, à maior demanda de fertilizantes. A análise de co-integração mostrou que as variáveis preço do fertilizante, crédito de custeio e índice de preço recebido apresentam uma raiz unitária. No entanto, a estimativa do modelo de correção de erros não se revelou satisfatória quanto ao nível de significância estatística dos coeficientes.

O consumo de fertilizantes no Brasil aumentou 680\% de 1970 a 2002, passando de 998 mil toneladas em 1970 para 7,77 milhões de toneladas em 2002. Constatou-se que, no longo prazo, a demanda de fertilizantes é inelástica a variações no seu preço e no volume de crédito rural e elástica em relação ao índice de preços recebidos pelos agricultores. Esses valores são coerentes com a essencialidade do produto para a agricultura brasileira e também são coerentes com estimativas feitas para outros países. A elasticidade-preço da demanda de fertilizantes no Brasil, no longo prazo, foi de $-0,655$ e a elasticidade cruzada em relação o volume de crédito rural foi de 0,250 . Essa inelasticidade em relação ao crédito rural oficial pode ser explicada, em parte, pelo fato dos agricultores usarem outras fontes de recursos para adquirirem esse insumo essencial ao processo de produção. Essas fontes de recursos são os recursos próprios e o de empresas que "compram" antecipadamente parte da produção, para assegurar o fornecimento do produto. 
O presente estudo estimou a demanda de fertilizantes como um todo para a agropecuária brasileira. Resultados diferentes podem surgir se forem feitas estimativas da demanda desse insumo por categorias de produtos agropecuários. Isto fica como sugestão para futuras pesquisas.

\section{Referências Bibliográficas}

AMARAL, F. C.S.; PEREIRA, N.R.; CARVALHO JUNIOR, W. Principais Limitações dos Solos Brasileiros. Empresa Brasileira de Pesquisa Agropecuária - EMBRAPA Solos, 1999. O tema é Solos. http//www.cnps. embrapa.br/search/pesqs/tema3/tema3.html . (03/04/2003).

ANDA - Anuário da Associação Nacional para Difusão de Adubos

BACCHI, M.R.P. Previsão de preços de bovino, suíno e frango com modelos de séries temporais. Piracicaba, 1994. 172p. Tese (Doutorado) - Escola Superior de Agricultura “Luiz de Queiroz”, Universidade de São Paulo.

BACEN - Anuário Estatístico do Credito Rural

BURREL,A. The demand for fertilizer in the UK. Journal of Agricultural Economics, v.40, p.1-20, 1989.

CIBANTOS J.S. Demanda de fertilizantes no Estado de São Paulo. Piracicaba, 1972, 196 p. Tese (Doutorado) - Escola Superior de Agricultura "Luiz de Queiroz", Universidade de São Paulo.

DENBALY, M.; VROOMEN,H. Dynamic fertilizer nutrient demands for corn: A cointegrated and error-correcting system. American Journal of Agricultural Economics, v.75, p.203-209, 1993.

DICKEY, D.A.; FULLER, W.A. Likelihood ratio statistics for autoregressive time series with a unit root. Econometrica, v.49, n.4, p.1057 - 1072, July 1981.

ENDERS, W. Applied econometric time series. New York: John Wiley, 1995. 433p.

ENGLE, R.F.; GRANGER, C.W.J. Co-integration and error correction: representation, estimation and testing. Econometrica, v.55, n.2, p.251276, Mar.1987.

ENGLE, R.F; YOO, B. Forecasting and testing in co-integrated systems. Journal of Econometrics. v.1, n.35, p.145-159, Maio 1987. 
GRILICHES, Z. Distributed lags, disaggregation, and regional demand function for fertilizer. Journal of Farm Economic, v.41, p.90-102, 1959.

GUILHOTO, J.J.M.; FURTUOSO, M.C.O.; BARROS, G.S.A.C. O Agronegócio na Economia Brasileira 1994 a 1999. Piracicaba: CEPEA/CNA, 2000. 139p.

HILL, R.C.;GRIFFTHS,W.E.;JUDGE,G.G. Econometria. São Paulo. Saraiva, $1999.408 p$.

IBGE- Anuário do Instituto Brasileiro de Geografia e Estatística.

JOHNSTON, J. Econometric Methods. New York. McGraw-Hill, 1972. 437p.

KAGEYAMA, A.; BUAINAIN, A.M.; REYDON, B.P.; SILVA, J.G.; SILVEIRA, J.M.; FONSECA, M.G.D.; RAMOS, P.; FONSECA, R.B.; BELIK, W. In: DELGADO, G.C.; GASQUES, J.G.; VERDE, C.M.V. (org.) Agricultura e políticas públicas. Brasília: IPEA, 1990.

MADDALA, G.S. Introduction to Econometric. Macmillan Publishing Company, New York: 1988.

MERGOS, G.J.; STOFOROS, Ch. E. Fertilizer demand in Greece. Agricultural Economics, v16, p.227-235, 1997.

NEVES, E.M.; RODRIGUES, L.; DAYOUB, M.; DRAGONE,D.S. Fertilizantes no Plano Real: estratégias empresariais e demanda. Piracicaba, 2002. 23p. Relatório de Pesquisa. Escola Superior de Agricultura “Luiz de Queiroz”, Universidade de São Paulo.

PESCARIN, R. M. C. Relações estruturais da demanda de fertilizantes no Estado de São Paulo, 1974. 123p. Dissertação(M.S.) - Escola Superior de Agricultura “Luiz de Queiroz”, Universidade de São Paulo.

SANTOS, H.G. Latossolos do Brasil. Empresa Brasileira de Pesquisa Agropecuária - EMBRAPA Solos, 1999. 0 tema é Solos http//www.cnps. embrapa.br/search/pesqs/tema1/tema1.html . (03/04/2003).

SOARES, A.C.M.; BARROS, J.R.M.; CARMO, A.J.B. Avaliação e perspectiva do comportamento da demanda de fertilizantes no Brasil. Revista de Economia Rural, v.21, n.1, p.29-60, jan/mar 1983. 